\title{
A Single Center retrospective five- year- experience of Extracorporeal Shock Wave Lithotripsy on
}

\section{Ureteral Stones: Outcomes and Recommendations}

\author{
George Nassar ${ }^{1}$, Mohamed J. Hejase ${ }^{2}$, Ale J. Hejase ${ }^{3}$, Hussin J. Hejase ${ }^{4, ~ * ~}$ \\ ${ }^{1}$ Urologist, Urolithiasis Specialist, Head of the Department of Urology, Saint Therese Hospital, Hadat, Lebanon. \\ ${ }^{2}$ Associate Urologist, Department of Urology, Saint Therese Hospital, Hadat, Lebanon. \\ ${ }^{3}$ Professor at the ITOM department, AKSOB, Lebanese American University, Beirut, Lebanon. \\ ${ }^{4}$ IEEE Senior Member, Al Maaref University, Management Science \& Technology, Beirut, Lebanon. \\ "Corresponding Author: Hussin Jose Hejase, IEEE Senior Member, Al Maaref University, Management Science \& Technology, PO box 5078/25 \\ Ghoubeiry, Beirut, Lebanon.
}

Received date: 16 July 2021; Accepted date: 26 July 2021; Published date: 30 July 2021

Citation: Nassar G, Hejase MJ, Hejase AJ, Hejase HJ. A Single Center retrospective five- year- experience of Extracorporeal Shock Wave Lithotripsy on Ureteral Stones: Outcomes and Recommendations. J Comm Med and Pub Health Rep 2(6): https://doi.org/10.38207/jcmphr20210097

Copyright: (C) 2021 Hussin J. Hejase. This is an open-access article distributed under the terms of the Creative Commons Attribution License, which permits unrestricted use, distribution, and reproduction in any medium, provided the original author and source are credited.

\begin{abstract}
Objective: This study aimed to recommend Extracorporeally Shock Wave Lithotripsy (ESWL) is the first choice in ureteral calculi. Evaluation of 1379 treatment sessions is used as supporting evidence.

Method: ESWL has been and still is one of the most common therapeutic means in the management of kidney and ureteral calculi. During a five-year period from 2009 -2013, 1754 ESWL session-treatments were performed using the 3000 H, Piezolith Lithotripter Wolf, Germany, at the Saint Therese Hospital Urology unit. 1379 treatment-sessions represented ureteral calculi. A cumulative percentage of $98.05 \%$ was reported treating 1085 out of 1103 after three-session treatments.

Results: The mean age of the patients was 44.13 years; $18.2 \%$ of the patients were females and $81.8 \%$ were males. The grand majority of the patients were outpatients (95.9\%). 1379 out of 1754 patient-cases had ureteral stones, 1085 out of 1103 (underwent a total of 3 sessions of ESWL). Distal ureteral calculi showed the best stone-free rates in $100 \%$, followed by mid ureteral calculi in $98.08 \%$, then by proximal ureteral stones in $94.83 \%$. Those results seem to be encouraging and satisfactory to the Urology unit's patients and to the urologists at the unit.

Conclusions: The large number of patient-cases, performed at a single medical center, with the same Lithotripter machine, and by the same physicians during the 5 year-period explains the successful results of fragmentation and stone clearance obtained. This paper asserts and advises Urology colleagues to stick to the Urinary Stone Management Guidelines and have ESWL as their first choice in Ureteral calculi treatment. Urologists should respect the inclusion and exclusion criteria, thus minimizing the complication rate and offering their patients the best results. Keywords: Extracorporeal shock wave lithotripsy; urology; ureteral stones; stone-free rates.
\end{abstract}

\section{Introduction}

In the past 25 years, the treatment of ureteral stones has evolved from Ureter lithotomy to Ureterorenoscopy URS, Extracorporeal shock wave lithotripsy and endoscopic lithotripsy. Following its clinical introduction by Chaussy et al. [1] in 1980 extracorporeal shock wave lithotripsy (ESWL) became the most common treatment modality with its safe and successful results in renal as well as in ureteral stones $[2,3]$. The unnecessary exposure to high energy shock waves may result in kidney, ureter, or neighboring tissue damage [4] if the treatment was not successful or well indicated. Urinary lithiasis can cause a greater or lesser degree of obstruction of the lower ureter and may need instrumentation such as Ureteroscopy and JJ stenting [5 - 8]. Regarding the parameters evaluated such as body mass index (BMI) [9, 10], skin to stone distance (SSD) [10], and stone related factors (stone location, diameter, and density in Hounsfield unit HU) $[\mathbf{1 0}, \mathbf{1 1}]$ if taken into consideration, would absolutely improve results
Concerning ureteral stones, which are our main interest in this article, have a high probability of spontaneous clearance. The spontaneous passage should be favored if possible [5 - 7]. According to the American Urological Association (AUA) guidelines, newly diagnosed ureteral stones smaller than $5 \mathrm{~mm}$ will pass spontaneously up to $98 \%$, depending on the degree of obstruction, urothelial edema, and degree of impaction $[\mathbf{5}, \mathbf{6}, \mathbf{1 2}, \mathbf{1 3}]$. Patients with ureteral stones would be tightly controlled considering the absence of risk factors such as urinary infection, fever, continuous pain, and impaired kidney function. A waiting period of 4-6 weeks should not be exceeded especially in case of obstruction. In this retrospective study, we aim to analyze our results of ESWL fragmentation rate on 1754 patientcases treated for their urinary stones, precisely interested in the ureteral stone cases. 


\section{Materials and Methods}

Between October 2009 and 2013 a total of 1754 patient-cases were received at our unit of Extracorporeal shock wave Lithotripsy using the Lithotripter (Piezolith 3000 Plus, Wolf, Germany) in the supine position (with variations depending on the stone location), having urinary stones in the kidney, ureter, or bladder, with a specific interest in ureteral stones. Patients with a solitary kidney, infected urine, renal failure, pregnant women, anticoagulation therapy (unless stopped for a week), stone impaction, and stones larger than $2 \mathrm{~cm}$ in diameter were excluded from the study. All patient-cases were confirmed with an unenhanced Abdominopelvic Computed Tomography NCCT, general blood, and coagulation tests, Urinalysis, and culture. The calculi were localized by Fluoroscopic guidance and Ultrasound. All patients were given sedative- analgesics and the level of shockwave energy (intensity) was progressively increased up till satisfactory fragmentation was obtained within the patient's comfort. The frequency, the number of shockwaves and the number of sessions given depended upon the stone's density, diameter and location,

\subsection{Ethical Considerations}

All work was conducted with the approval of an ethics committee (Saint Therese Hospital, Department of Urology, PO box 169, Hadrat, Beirut, Lebanon).

Patients were informed that their ESWL records are confidentially kept in their personal file [either at the hospital or their immediate attending medic]. In the case of using their data, no personal identities or personal information is revealed whatsoever. All results will be used selectively based on the subject of the academic/professional research performed.

All patients were received and attended with all possible comfort and attention since the first visitation and through the treatment process

\section{Results}

The data collected corresponds to 1754 lithotripsy procedures performed within the time frame from March 26, 2009, till October 28,2013 . The demographics characteristics of the patients are as follows: the mean age of the patients was 44.13 years (Standard Deviation $=14.275), 18.2 \%$ of the patients were females $($ mean age $=$ 46.61years), and $81.8 \%$ were males (mean age $=43.57$ years) Likewise, the grand majority of the patients were outpatients (95.9 $\%)$. The health providers were the Lebanese armed forces (36.7\%), National Soc. Sec. - CNSS (30.73\%), and CNSS + Private [Maladies] $(5.82 \%)$, and $53 \%, 46.10 \%$, and $0.9 \%$ of the extracorporeal shock wave lithotripsy (ESWL) were done on the left, right sides, and the bladder, respectively of the patients.

Moreover, the distribution of age for both the female and male patients, respectively are nearly normal. Mann-Whitney U test for two independent samples was performed to test if the average ages of the patients are different between females and males. Results revealed at $5 \%$ level of significance ( $95 \%$ confidence) that the distributions of knowing that patients who had a low pain threshold might have needed a double $\mathrm{J}$ stent placement under anesthesia prior to the procedure. Complete stone clearance was assessed at a 3-month follow-up. Plain $X$ rays (patients with radiolucent stones needed Ultrasound or Tomographic cuts) were taken at 3- and 6-week intervals to check for stone fragments and their clearance, success, or complications in order to decide if the patient needed subsequent sessions or not. If the patients continued with flank pain, or ureteral colic after a 2-month period, another treatment modality was implemented. Treatment failure was based on the need for further surgical or endoscopic interventions or failure to become stone-free after 3 months. Data were analyzed using the Statistical Product and Service Solutions, SPSS version 25 [14]. Both univariate (Pearson's Chi-square and Student t-Test), and the non-parametric tests namely Mann-Whitney U-test, Spearman correlation test, and Kruskal Wallis test was used to determine the correlations wherever appropriate, and $\mathrm{p}<0.05$ was considered statistically significant.

and the final curing. At any time, patients were kept relaxed, conscientious about the details of the process, and were informed to continuously provide their state of mind.

Patients were made aware with pertinent information and advice, after defining the indications of treatment, of both the modalities of treatment and their probable complications. In fact, the following items were explained to the patient: "The need for anesthesia, stent, urethral manipulation, possible complications, need for repeated follow up especially after ESWL, and the cost factor involved." The mode of treatment was the patients' choice.

ages between females and males are significantly different $(\mathrm{p}$-value $=$ 0.002) whereas, in general, the distribution of ages of females undergoing lithotripsy procedures are higher than those ages of males undergoing similar procedures. To reinforce the fact that within lithotripsy procedures, female ages are generally higher than male ages, an independent sample t-test was performed. This test which requires that each of the samples must follow a normal distribution (which is true in the current study) disclosed that the averages of both groups are in fact different ( $\mathrm{p}$-value $=0.003)$.

Furthermore, testing the distribution of lithotripsy procedure sessions applied for both the female and male patients respectively was performed. Results reveal that both sessions distributions are nearly the same (average for males 1.35 sessions and for females 1.36 sessions). To emphasize such a conclusion, a Mann-Whitney $U$ test for two independent samples was performed and it revealed that at a $5 \%$ level of significance (95\% confidence) the distributions of a number of sessions for females and males are not significantly 
different ( $p$-value $=0.514$ ); thus, the distributions of the number of lithotripsy sessions across both gender categories are the same. On asking if "there is any relation or dependency between the gender of the patient and the side where the lithotripsy session took place?" A chi-square test of independence was performed, and the outcome was $\chi^{2}=4.119$ with $\mathrm{p}$-value $=0.127$ indicating independence between gender and the side of the lithotripsy session. Actually, the data show that $55.3 \%$ of the females and $52.5 \%$ of the males did go through the lithotripsy session on the left side; likewise, $44.7 \%$ of the females and $46.4 \%$ of the males did go through the lithotripsy session on the right side and the aforementioned left and right percentages are not significantly different.

\subsection{ESWL Treatment Results}

Table 1: The frequencies of stone free results under the different session numbers

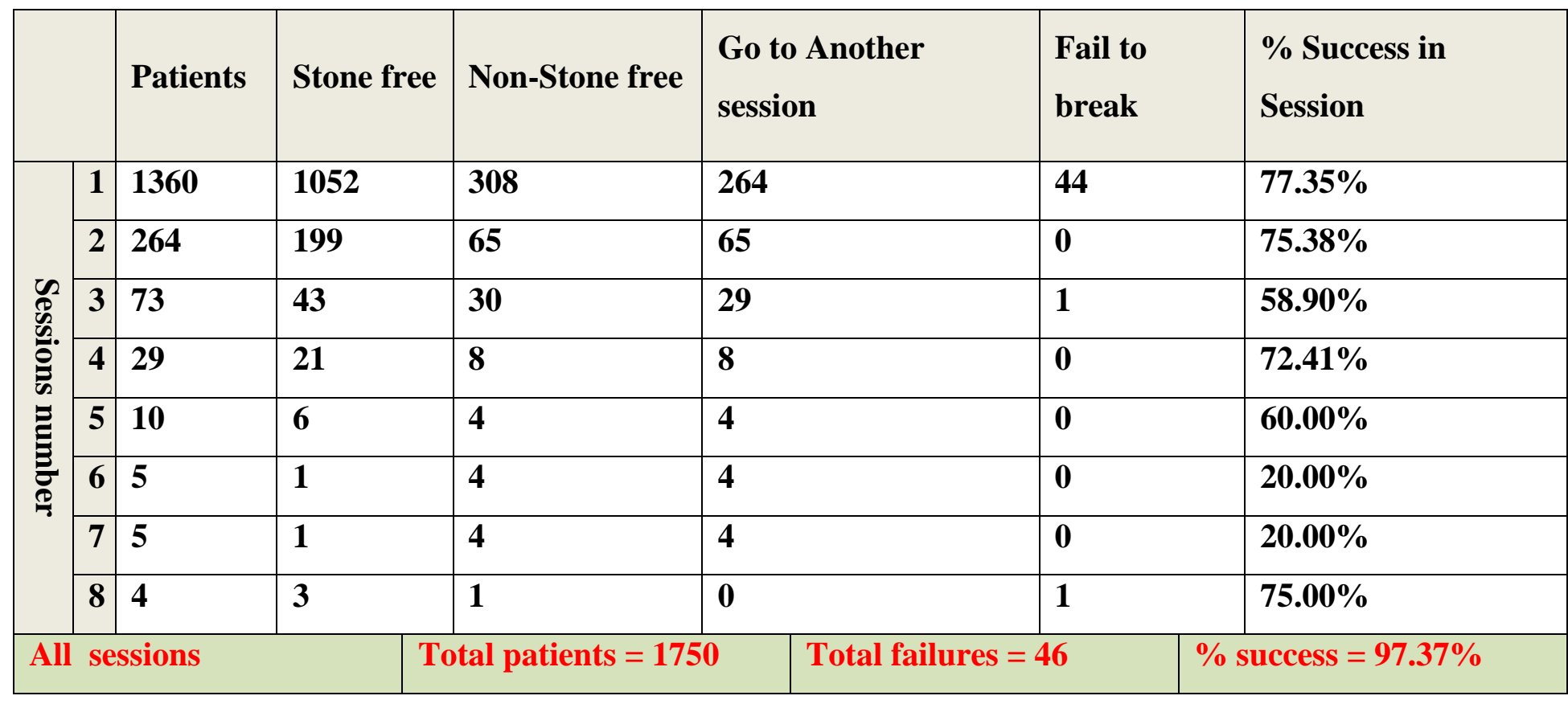

Table 1 shows that the number of patients that did the ESWL treatment for the first time is 1360; out of which the shock waves were able to break up the stones in 1052 cases (success rate $77.35 \%$ ). Out of the 308 non-stone free cases, 264 went for the second treatment indicating that in 44 cases the shock waves failed to break the stones.

\subsubsection{Stone location effect}

Next, it is worth testing if there is any dependency between the result of the treatment and the stone location side. For that purpose, a chisquare test of independence was carried out and it resulted in a $\chi^{2}=$ 8.467 with $p$-value $=0.015$ indicating a significant dependence (at $5 \%$

\subsubsection{Stone density effect}

As for the stone density effect, Table 2 displays the number of cases for each stone density category. It is clear from the table that bone density is the most common (52.34\% of the cases) followed by radiolucent and uric acid stones (30.14\% of the cases). Moreover, the authors raised another inquiry specifically, "Is there any relation between the patient's age and the stone density?" The answer to such question is supported by calculating the nonparametric Spearman coefficient of correlation which provided no significant correlation value, though statistically valid, between the aforementioned variables (Spearman coefficient of correlation equal to 0.068 with a p-value equal to 0.005). Moreover, the chi-square test of independence between stone density and gender gave a $\chi 2=5.36$ with p-value $=0.069$ demonstrating that the stone densities are independent of gender (at a $5 \%$ level of significance); however, at a
In the second session, the success rate was almost the same (75.38 $\%$ ). Additionally, Table 1 reveals that for the total of 1750 treatments, only 46 cases ended with a negative stone-free result (1704 stone free cases) thus reflecting a general success rate of $97.37 \%$.

level of significance) between the result of the test (stone-free) and the stone location side (left/right) where it looks that the percentage of failures for all the sessions is higher on the left side $(25.9 \%)$ than that on the right side $(21.6 \%)$.

$10 \%$ level of significance, the dependency does arise. Furthermore, the authors studied if the age distributions are the same across the three different categories of stone densities? Here, the nonparametric Kruskal-Wallis test was used to verify if the distributions of different independent groups are identical. The chi-square value of the Kruskal-Wallis test resulted in 11.044 with a p-value of 0.004 , thus indicating that the distributions of age are different among the threestone density categories. In fact, the average age for bone density was 45 years (median $=44$ years and standard deviation $=14.135$ years), likewise, for medium density the average was 42.83 years (median = 40 years and standard deviation $=14.301$ ) and for radiolucent density the average age was 43.38 years (median $=42$ years and standard deviation $=14.444$ years). 
Table 2: Frequencies and percentages of stone densities in the treatments of this study

\begin{tabular}{|l|l|l|l|}
\hline & Frequency & Percent & Cumulative Percent \\
\hline Radiolucent & 528 & $30.14 \%$ & $30.14 \%$ \\
\hline Medium & 307 & $17.52 \%$ & $47.66 \%$ \\
\hline Bone density & 917 & $52.34 \%$ & $100.00 \%$ \\
\hline Total & 1752 & $100.00 \%$ & \\
\hline
\end{tabular}

\subsubsection{Distribution of the location and side of the patient's treated stones}

Table 3 depicts the results of the details pertaining to the location and size of the patient's treated stones. Table $\mathbf{3}$ shows that the dominant stones are mainly left and right distal ureteral stones with the left percentage $(26 \%)$ exceeding that of the right $(23 \%)$.

Table 3: Distribution of stones according to location and side

\begin{tabular}{|c|c|c|c|}
\hline No. & Side and location & Frequency & Percent \\
\hline 1 & Left distal ureteral stone & 381 & $27.02 \%$ \\
\hline 2 & Right distal ureteral stone & 324 & $22.98 \%$ \\
\hline 3 & Right proximal ureteral stone & 117 & $8.30 \%$ \\
\hline 4 & Left proximal ureteral stone & 115 & $8.16 \%$ \\
\hline 5 & Left renal pelvis stone & 98 & $6.95 \%$ \\
\hline 6 & Right renal pelvis stone & 82 & $5.82 \%$ \\
\hline 7 & Left mid ureteral stone & 81 & $5.74 \%$ \\
\hline 8 & Right mid ureteral stone & 75 & $5.32 \%$ \\
\hline 9 & Right upper calyceal stone & 20 & $1.42 \%$ \\
\hline 10 & Right lower calyceal stone & 16 & $1.13 \%$ \\
\hline 11 & Left lower calyceal stone & 11 & $0.78 \%$ \\
\hline 12 & Left upper calyceal stone & 11 & $0.78 \%$ \\
\hline 13 & Left renal pelvis, upper and lower calyceal stones & 4 & $0.28 \%$ \\
\hline 14 & Left renal pelvis and lower calyceal stones & 3 & $0.21 \%$ \\
\hline 15 & Left renal pelvis and proximal ureteral stones & 3 & $0.21 \%$ \\
\hline 16 & Right renal pelvis and upper calyceal stones & 2 & $0.14 \%$ \\
\hline 17 & Bilateral multiple staghorn kidney stones & 1 & $0.07 \%$ \\
\hline 18 & Left distal ureteral steinstrasse & 1 & $0.07 \%$ \\
\hline 19 & Left proximal ureteral stones and renal pelvis stone & 1 & $0.07 \%$ \\
\hline 20 & Left renal pelvis and upper calyceal stones & 1 & $0.07 \%$ \\
\hline 21 & Left renal pelvis stone (solitary kidney) & 1 & $0.07 \%$ \\
\hline 22 & Left staghorn renal pelvis stone & 1 & $0.07 \%$ \\
\hline 23 & Left ureteral stones & 1 & $0.07 \%$ \\
\hline 24 & Right renal pelvis and calyceal stone & 1 & $0.07 \%$ \\
\hline 25 & Right renal pelvis and mid calyceal stones & 1 & $0.07 \%$ \\
\hline 26 & Right upper and lower calyceal stones & 1 & $0.07 \%$ \\
\hline 27 & Upper calyceal stone & 1 & $0.07 \%$ \\
\hline 28 & Urinary bladder stone & 9 & $0.64 \%$ \\
\hline 29 & Left proximal ureteral stone with double $\mathrm{J}$ & 6 & $0.43 \%$ \\
\hline 30 & Left mid ureteral stone with double $\mathrm{J}$ & 4 & $0.28 \%$ \\
\hline 31 & Left distal ureteral stone with double $\mathrm{J}$ & 15 & $1.06 \%$ \\
\hline 32 & Right distal ureteral stone with double J & 9 & $0.64 \%$ \\
\hline 33 & Right proximal ureteral stone with double $\mathrm{J}$ & 9 & $0.64 \%$ \\
\hline \multirow[t]{2}{*}{34} & Right mid ureteral stone with double $\mathrm{J}$ & 4 & $0.28 \%$ \\
\hline & Total & 1410 & $100.00 \%$ \\
\hline
\end{tabular}


Moreover, the first ten rows of Table 3 show that $92.284 \%$ of the stones are characterized as follows: Left distal ureteral stone (27.02\%), Right distal ureteral stone (22.98\%), Right proximal

\subsubsection{Single stone treatment cases}

Table 4 presents the sizes of the stones identified for those patients who suffered from a single stone where it is seen that the most common stone size is the $1 \mathrm{~cm}$ stone $(23.63 \%$ of the cases) followed ureteral stone $(8.30 \%)$, Left proximal ureteral stone $(8.16 \%)$, Left renal pelvis stone (6.95\%), Right renal pelvis stone (5.82\%), Left mid ureteral stone (5.74\%) and Right mid ureteral stone (5.32\%).

by the $0.8 \mathrm{~cm}$ and $0.7 \mathrm{~cm}$ with percentages of $16.15 \%$ and $15.41 \%$, respectively.

Table 4: Stone sizes and percentages for single stone treatments

\begin{tabular}{|c|c|c|c|c|}
\hline & Stone $(\mathrm{cm})$ & Number of cases & Percent & Cumulative Percent \\
\hline \multirow{34}{*}{ 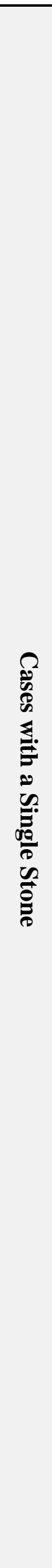 } & 0.20 & 3 & $0.17 \%$ & $0.17 \%$ \\
\hline & 0.40 & 2 & $0.11 \%$ & $0.29 \%$ \\
\hline & 0.45 & 1 & $0.06 \%$ & $0.34 \%$ \\
\hline & 0.50 & 69 & $3.94 \%$ & $4.28 \%$ \\
\hline & 0.60 & 159 & $9.08 \%$ & $13.36 \%$ \\
\hline & 0.70 & 270 & $15.41 \%$ & $28.77 \%$ \\
\hline & 0.75 & 2 & $0.11 \%$ & $28.88 \%$ \\
\hline & 0.80 & 283 & $16.15 \%$ & $45.03 \%$ \\
\hline & 0.90 & 169 & $9.65 \%$ & $54.68 \%$ \\
\hline & 1.00 & 414 & $23.63 \%$ & $78.31 \%$ \\
\hline & 1.10 & 22 & $1.26 \%$ & $79.57 \%$ \\
\hline & 1.20 & 80 & $4.57 \%$ & $84.13 \%$ \\
\hline & 1.30 & 41 & $2.34 \%$ & $86.47 \%$ \\
\hline & 1.40 & 7 & $0.40 \%$ & $86.87 \%$ \\
\hline & 1.50 & 63 & $3.60 \%$ & $90.47 \%$ \\
\hline & 1.60 & 7 & $0.40 \%$ & $90.87 \%$ \\
\hline & 1.70 & 5 & $0.29 \%$ & $91.15 \%$ \\
\hline & 1.80 & 10 & $0.57 \%$ & $91.72 \%$ \\
\hline & 2.00 & 44 & $2.51 \%$ & $94.24 \%$ \\
\hline & 2.20 & 1 & $0.06 \%$ & $94.29 \%$ \\
\hline & 2.30 & 5 & $0.29 \%$ & $94.58 \%$ \\
\hline & 2.50 & 14 & $0.80 \%$ & $95.38 \%$ \\
\hline & 2.70 & 1 & $0.06 \%$ & $95.43 \%$ \\
\hline & 2.80 & 2 & $0.11 \%$ & $95.55 \%$ \\
\hline & 3.00 & 7 & $0.40 \%$ & $95.95 \%$ \\
\hline & 3.50 & 3 & $0.17 \%$ & $96.12 \%$ \\
\hline & 3.70 & 1 & $0.06 \%$ & $96.18 \%$ \\
\hline & 4.00 & 4 & $0.23 \%$ & $96.40 \%$ \\
\hline & 4.40 & 1 & $0.06 \%$ & $96.46 \%$ \\
\hline & 4.50 & 1 & $0.06 \%$ & $96.52 \%$ \\
\hline & 5.00 & 1 & $0.06 \%$ & $96.58 \%$ \\
\hline & 7.00 & 2 & $0.11 \%$ & $96.69 \%$ \\
\hline & 8.00 & 3 & $0.17 \%$ & $96.86 \%$ \\
\hline & 9.00 & 1 & $0.06 \%$ & $96.92 \%$ \\
\hline & Total & 1698 & $96.92 \%$ & \\
\hline
\end{tabular}




\subsubsection{Multiple stones treatment cases}

Similarly, Table 5 presents the sizes of the stones identified for those patients who suffered from multiple stones. It is worth mentioning that Tables 4 and 5 demonstrate that the percentage of cases with a

Table 5: Stone sizes and percentages for multiple stone treatments single stone are $96.92 \%$ while the 54 multiple stones cases form only $3.08 \%$ of the total number of treatments.

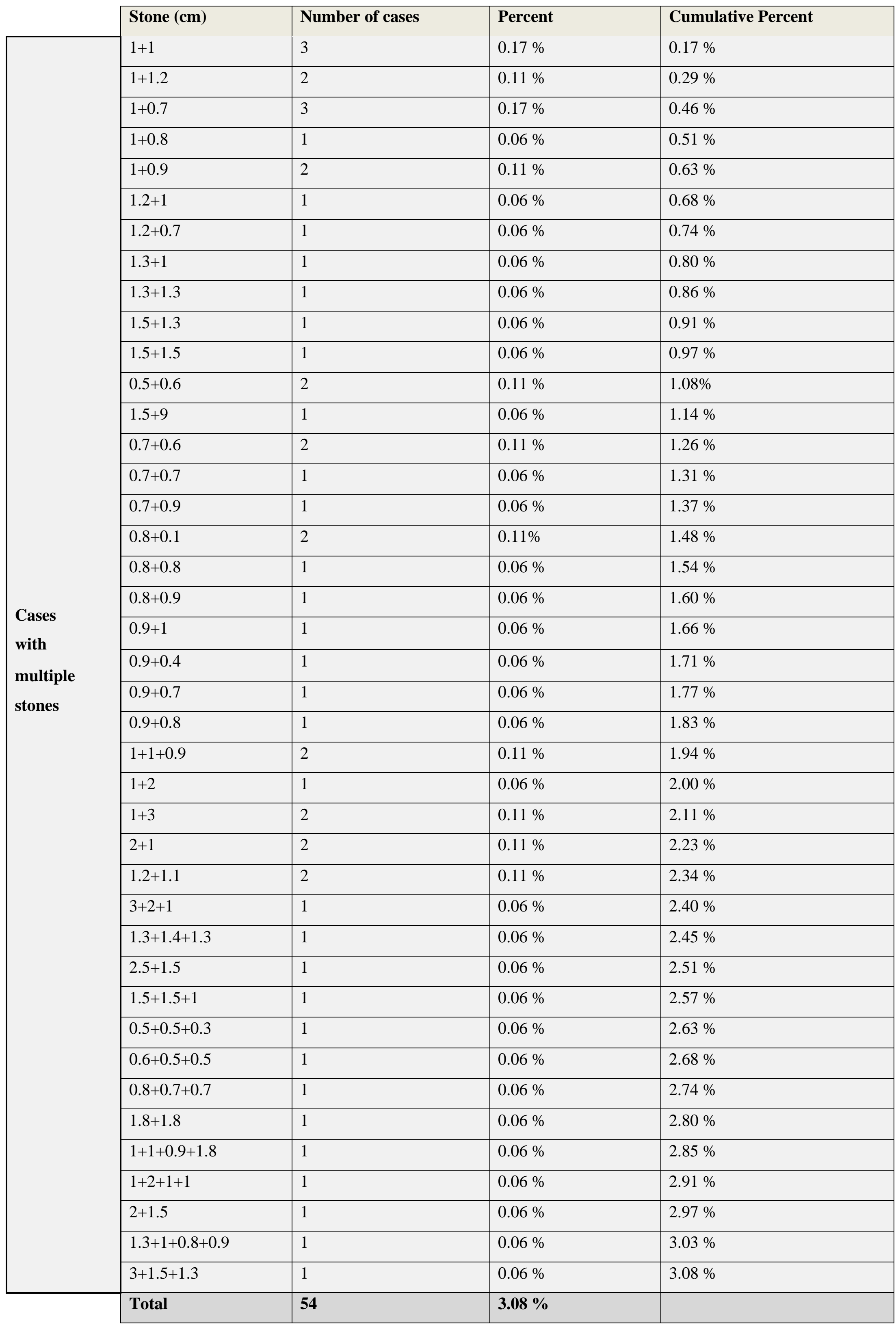


Next, the following question is addressed: "Are the medians and distributions of stone sizes the same across both genders?" For this purpose, the nonparametric independent samples Mann-Whitney U test was performed. The result shows that there is no statistically significant difference between the distributions of stone sizes between the two gender categories ( $\mathrm{p}$-value $=0.354$ ). Moreover, the independent samples Median Test concluded that the median stone size is the same for both gender categories ( $p$-value $=0.272$ ). Additionally, on querying if stone size and age are related; the nonparametric Spearman coefficient of correlation was used to deduce that its value is 0.146 with a p-value of 0.000 indicating a very low value, though statistically significant, correlation.

\subsubsection{Pulse counts for the total treatments}

Table 6 shows the statistics for the pulse counts used in the treatments where it is clearly illustrated that the average pulse count is around 4400 pulses with a standard deviation of around 1500 pulses. Notice that the mode is 4000 pulses making the 4000 pulses the most

common setup for lithotripsy. 388 treatments out of 1754 were performed with a setup of 4000 pulses (this represents $22.12 \%$ of the cases), $358(20.41 \%)$ treatments were done using 5000 pulses, and $253(14.42 \%)$ treatments were performed using 6000 pulses.

Table 6: Statistics on the pulse counts for the 1754 treatments

\begin{tabular}{|l|l|}
\hline Mean & 4403.461 \\
\hline Median & 4000.000 \\
\hline Mode & 4000.0 \\
\hline Std. Deviation & 1535.8802 \\
\hline Variance & 2358928.063 \\
\hline Skewness & 0.371 \\
\hline Std. Error of Skewness & 0.058 \\
\hline Kurtosis & 0.946 \\
\hline Std. Error of Kurtosis & 0.117 \\
\hline Range & 13000.0 \\
\hline Minimum & 1000.0 \\
\hline Maximum & 14000.0 \\
\hline
\end{tabular}

Moreover, when discussing the issue of lithotripsy pulse count, a researcher might ask: "Is the stone density a determinant factor in the pulse count selection?" The Spearman coefficient of correlation may provide an insight into the question. Results show that there is a statistically significant linear high positive relation between pulse counts and stone densities (coefficient of correlation equal to 0.616 with a p-value equal to 0.000$)$. As for the relation between pulse count and stone size, the nonparametric Spearman coefficient of correlation resulted in a value of 0.391 ( $\mathrm{p}$-value $=0.000$ ) implying a statistically significant moderate positive linear relation between pulse count and stone size. In order to perform a more realistic analysis that provides results of ureteral stones fragmentation, Tables 7 and 8 considered exclusively the ureteral stones locations and outcomes.

Table 7: Treatment outcome up to three ESWL sessions

\begin{tabular}{|c|c|c|c|}
\hline & $\begin{array}{l}\text { Treatments that started in session } \\
1\end{array}$ & Stone free after 3 sessions & Stone free \\
\hline & Count & Count & $\begin{array}{l}\text { Rate after } 3 \\
\text { sessions }\end{array}$ \\
\hline Right proximal ureteral stone & 115 & 109 & $94.78 \%$ \\
\hline Right proximal ureteral stone with double J & 9 & 8 & $88.89 \%$ \\
\hline Left proximal ureteral stone & 115 & 110 & $95.65 \%$ \\
\hline Left proximal ureteral stone with double $\mathbf{J}$ & 6 & 6 & $100.00 \%$ \\
\hline Right mid ureteral stone & 73 & 72 & $98.63 \%$ \\
\hline Right mid ureteral stone with double $\mathbf{J}$ & 4 & 4 & $100.00 \%$ \\
\hline Left mid ureteral stone & 79 & 74 & $93.67 \%$ \\
\hline
\end{tabular}




\begin{tabular}{|c|c|c|c|}
\hline Left mid ureteral stone with double $\mathbf{J}$ & 4 & 4 & $100.00 \%$ \\
\hline Right distal ureteral stone & 313 & 320 & $100.00 \%$ \\
\hline Right distal ureteral stone with double J & 8 & 9 & $100.00 \%$ \\
\hline Left distal ureteral stone & 363 & 371 & $100.00 \%$ \\
\hline Left distal ureteral stone with double $\mathbf{J}$ & 14 & 14 & $100.00 \%$ \\
\hline Totals & 1103 & 1101 & $\mathbf{9 9 . 8 2 \%}$ \\
\hline
\end{tabular}

Lithotripsy takes place through a series of treatment sessions. In most of the cases (909 out of 1103, i.e., $82.4 \%$ ) a stone-free result is achieved after the first session. Those cases where the stone-free fails are taken to posterior successive sessions that in very rare occasions $(0.18 \%)$ reach session number 8 . Table 7 depicts the number of case treatments for the different ureteral stone locations and sides that were started with session 1 . The same table shows for each ureteral location and sides the number of successful treatments after the completion of three sessions. For example, for the right mid ureteral stones, 73 treatment cases were started, and 58 cases were recorded stone-free; the remaining 15 cases were taken to another posterior session (session 2) then again, the failing cases were taken to session 3. In brief as Table 7 shows, after three sessions the 73 original cases ended up with 72 stone-free successes with only one case to be taken forward to a fourth session. This represents a $98.63 \%$ success rate which is accomplished after terminating the third session. Similarly, Table 7 shows that the success rate for left proximal ureteral stones is $95.65 \%$ which is achieved after three sessions where the initial 115 cases had 78 successes in the first session, the remaining 37 went to session 2 then session 3 for the failures to end up with only 5 cases thus accomplishing a success rate of $95.65 \%$. In addition, the bottom row of Table 7 shows that for the initial 1103 treatments, 1101 were recorded as stone-free (success rate $99.82 \%$ ) where it is worth mentioning that during the transitions from session 1 to session 3 some patients did abandon the treatment while others did join after they have undergone earlier treatments in other places.

Table 8: Detailed explanation for the success percentages.

\begin{tabular}{|c|c|c|c|c|c|}
\hline \multirow{2}{*}{$\begin{array}{l}\text { Session } \\
\text { number }\end{array}$} & \multicolumn{2}{|l|}{ Results } & \multirow{2}{*}{$\begin{array}{l}\text { Cumulative } \\
\text { Stone Free }\end{array}$} & \multirow{2}{*}{$\begin{array}{l}\text { Cumulative \% } \\
\text { Stone Free }\end{array}$} & \multirow{2}{*}{$\begin{array}{l}\text { Total } \\
\text { Treatments }\end{array}$} \\
\hline & Failed & Stone Free & & & \\
\hline 1 & $194(17.6 \%)$ & $909(82.41 \%)$ & 909 & $80.87 \%(909 / 1124)$ & 1103 \\
\hline 2 & $33(16.8 \%)$ & $164(83.2 \%)$ & 1073 & $95.46 \%(1073 / 1124)$ & 197 \\
\hline 3 & $18(38.3 \%)$ & $29(61.7 \%)$ & 1102 & $98.045(1102 / 1124)$ & 47 \\
\hline 4 & $5(25.0 \%)$ & $15(75.0 \%)$ & 1117 & $99.38 \%(1117 / 1124)$ & 20 \\
\hline 5 & $1(16.7 \%)$ & $5(83.3 \%)$ & 1122 & $99.82 \%(1122 / 1124)$ & 6 \\
\hline 6 & $2(100.0 \%)$ & $0(0.0 \%)$ & 1122 & $99.82 \%(1122 / 1124)$ & 2 \\
\hline 7 & $2(100.0 \%)$ & $0(0.0 \%)$ & 1122 & $99.82 \%(1122 / 1124)$ & 2 \\
\hline 8 & $0(0.0 \%)$ & $2(100.0 \%)$ & 1124 & $100.0 \%(1124 / 1124)$ & 2 \\
\hline Total & 255 & 1124 & 1124 & & 1379 \\
\hline
\end{tabular}

Table 8 shows the success rate per session together with the cumulative success stone free rate. Specifically, after the first session where 1103 patients were treated, 909 cases resulted in stone free $(82.41 \%)$; likewise, after session 2, the successful cases were 164 out of 197 i.e., $83.25 \%$ (here it is worth noticing that 3 new patients entered at session 2 having done session 1 elsewhere). The cumulative success rate after the two initial sessions are $95.46 \%$ obtained from
909 plus 164 successful cases that were marked as stone-free divided by the total number of all cases which is 1124 (Recall that to the initial 1103 patients we have added the new entrants and subtracted those that did abandon the treatment being now a total of 1124 cases). Finally, Figure 1 is a graphical equivalent of Table 8 which clearly shows the successful stone-free rate for each class of right and left ureteral stones. 


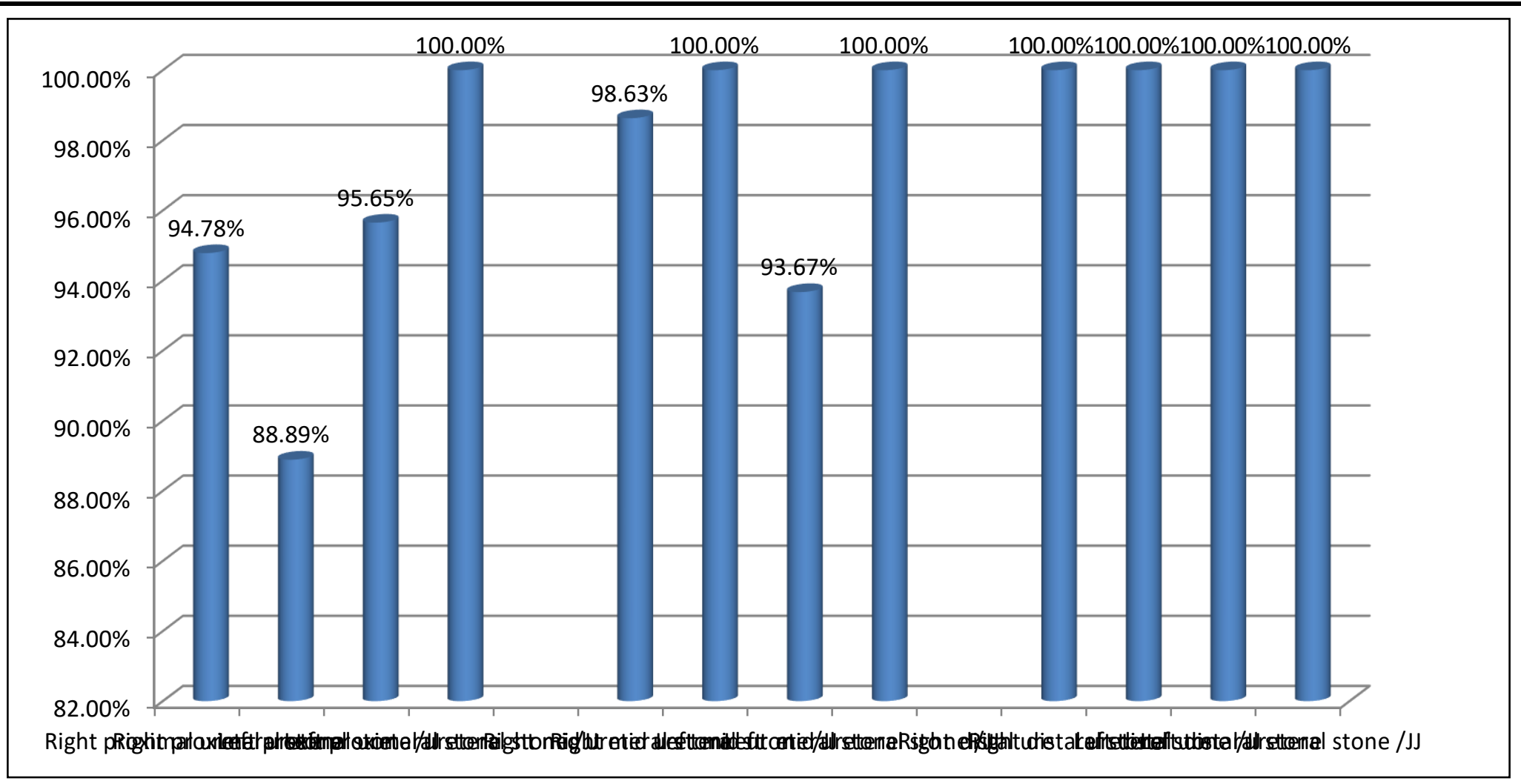

Figure 1. Bar Graph for the stone free rates

\section{Discussion}

ESWL has been established by urologists worldwide as the frontline therapeutic method for renal and ureteric calculi since the early $80 \mathrm{~s}$ $[2,3]$. The advent of new techniques in stone treatment such as Ureteroscopy, PCNL, and RIRS have badly tried to push aside the ESWL, but up till now, that has not been possible. In fact, many recent research results are not out, and the current status is that each method has its pros and cons [15 - 18]. At Saint Therese Hospital, the first two authors use the ureteroscopy modality for stones that cannot be cleared with ESWL, since ESWL is being their first choice to fragment ureteral stones. ESWL is well known to be less invasive than all other modalities as a stone treatment since it does not need anesthesia, neither needs surgical instrumentation, gas, and fluid insufflation nor incapacitating incisions [5 - 7]. If the inclusion criteria were well respected, the results of the total given sessions would absolutely satisfy the patient and the treating Urologist. The overall fragmentation rate of ureteric stones using ESWL has been reported in several series to range between 84 and $96 \%$, with and without the use of alpha1 blockers and diuretics $[8,12,19]$. In our studied series, 1379 out of 1754 patient-cases had ureteral stones,

\section{Conclusions}

The large number of patient cases, performed at a single medical center, with the same Lithotripter-machine and by the same physicians during the 5 year-period explain the successful results of fragmentation and stone clearance obtained. We highly recommend our colleagues to tightly attach to the AUA guidelines and try to be as noninvasive as possible upon managing their patients. This recommendation is congruent with those of Reynolds, Korczak, and
1085 out of 1103 (underwent a total of 3 sessions of ESWL), with an $80.87 \%$ stone free success rate for the first session. As to the second session 164 out of 197 treatments resulted in stone-free $95.46 \%$. For the third session, 29 out of 47 treatments resulted in a cumulative stone free percentage of $98.05 \%$ (Table 8). As depicted in our cumulative percentage tables, distal ureteral stone treatments had the best results of $100 \%$, followed by the mid ureteral stones with 98.08 $\%$, and then by the proximal ureteral stones with $94.83 \%$ (Table 7), and that is due to the physiology and anatomy of the ureter during an ESWL session, and the shorter distance stone fragments would travel to reach out for the ureteral orifice [20 - 23]. According to Pettersson \& Tiselius [23], lower or distal ureteral stones had a better fragmentation rate of $100 \%$ compared to proximal ones $93 \%$, results comparable to ours. In other series, success rates ranged from $79 \%$ in proximal ureter calculi, $78 \%$ in mid ureter calculi, and $79 \%$ in distal ureter calculi. Predictors of success are size, location, composition-density, and renal or ureteral anatomy. Obesity, skin to stone distance, and patient compliance during and after the treatmentsession may affect the outcome $[\mathbf{2 3}, \mathbf{2 4}]$.

Pace [25]. There have been many disputes and discrepancies concerning the complications an ESWL might cause to the urinary tract, but those were much lesser than the complications caused by other modalities since the inclusion and exclusion criteria were well respected. We still recommend ESWL as the first choice in the management of ureteral stones since the outcomes are satisfactory and encouraging to us as well as to our patients. 


\section{Author contributions}

Study concept and design: George Nassar, Mohamed J. Hejase, Ale J. Hejase,

Generation and collection of data: George Nassar, Mohamed J.

Hejase

Assembly, data cleansing and validation: George Nassar, Mohamed

J. Hejase, Ale J. Hejase, Hussin Hejase

Statistical analysis: Ale J. Hejase, Hussin Hejase

7. Conflict of Interest Statement: The authors report no conflicts of interest.

\section{References}

1. Chaussy C, Scmiedt E, Jocham D, Brendel W, Forssmann B, et al. (1982) First clinical experience with extracorporeally induced destruction of kidney stones by shock waves. J Urol. 127(3): 417 20.

2. Ghalayini IF, Al-Ghazo MA, Khader YS (2008) Evaluation of emergency extracorporeal shock wave lithotripsy for obstructing ureteral stones. Int Braz J Urol. 34(4): 433-40.

3. Motola JA, Smith AD (1990) Therapeutic options for the management of upper tract calculi. Urol Clin North Am. 17(1): 191-206.

4. Yazici O, Tuncer M, Sahin C, Demirkol MK, Kafkasli A, et al. (2015) Shock Wave Lithotripsy in Ureteral Stones: Evaluation of Patient and Stone Related Predictive Factors. Int Braz J Urol. 41(4): 676-682.

5. Abdelghany M, Zaher T, El Halaby R, Osman T (2011) Extracorporeal shock wave lithotripsy of lower ureteric stones: Outcome and criteria for success. Arab J Urol. 9(1): 35-9.

6. Segura JW, Preminger GM, Assimos DG, Dretler SP, Kahn RI, et al. (1997) Ureteral stones clinical guidelines panel summary report on the management of ureteral calculi. J Urol. 158(5): 1915-1921.

7. Abdel Khalek M, Sheir K, Elsobky E, Showkey S, Kenawy M (2003) Prognostic factors for Extracorporeal Shock wave Lithotripsy of ureteric stones-a multivariate analysis study. Scand J Urol Nephrol. 37(5): 413-418.

8. Tiselius HG (1993) Anesthesia-free extracorporeal shock wave lithotripsy of distal ureteral stones without a ureteral catheter. J Endourol. (4): 285-7.

9. Carbone A, Al Salhi Y, Tasca A, Palleschi G, Fuschi A, et al. (2018) Obesity and kidney stone disease: a systematic review. Minerva Urol Nefrol. 70(4): 393-400.

10. Pareek G, Hedican SP, Lee FT Jr, Nakada SY (2005) Shock wave lithotripsy success determined by skin-to-stone distance on computed tomography. Urology. 66(5): 941-944.
Analysis and interpretation: George Nassar, Mohamed J. Hejase, Ale J. Hejase, Hussin Hejase

Drafting the manuscript: George Nassar, Mohamed J. Hejase, Ale J. Hejase, Hussin Hejase

Critical revision of the manuscript for important intellectual content: George Nassar, Mohamed J. Hejase, Ale J. Hejase, Hussin Hejase Final approval of the manuscript: George Nassar, Mohamed J.

Hejase, Ale J. Hejase, Hussin Hejase

8. Declaration of Conflicting Interests: The author(s) declared no potential conflicts of interest with respect to the research, authorship, or publication of this article.

11. Gücük A, Uyetürk U (2014) Usefulness of hounsfield unit and density in the assessment and treatment of urinary stones. World J Nephrol. 3(4): 282-286.

12. Hochreiter WW, Danuser H, Perrig M, Studer UE (2003) ESWL for distal ureteral calculi: what a powerful machine can achieve. $\mathrm{J}$ Urol. 2003; 169(3): 878-880.

13. Mokhless I, Zahran AR, Youssif M, Fouda K, Fahmy A (2012) Factors that predict the spontaneous passage of ureteric stones in children. Arab J Urol. 10(4): 402-407.

14. Hejase AJ, Hejase HJ. Research Methods, A Practical Approach for Business Students; 2nd edition, Masadir Inc., Philadelphia, PA, 2013.

15. Guyatt G, Oxman AD, Akl EA, Kunz R, Vist G, et al. (2011) GRADE guidelines: 1. Introduction-GRADE evidence profiles and summary of findings tables. J Clin Epidemiol. 64(4): 383-94.

16. Egger M, Davey Smith G, Schneider M, Minder C (1997) Bias in meta-analysis detected by a simple, graphical test. BMJ. 315: $629-34$.

17. Srisubat A, Potisat S, Lojanapiwat B, Setthawong V, Laopaiboon M (2014) Extracorporeal shock wave lithotripsy (ESWL) versus percutaneous nephrolithotomy (PCNL) or retrograde intrarenal surgery (RIRS) for kidney stones. Cochrane Database Syst Rev. 11: CD007044.

18. Cao L, Wang YQ, Yu T, Sun Y, He J, et al. (2020) The effectiveness and safety of extracorporeal shock wave lithotripsy for the management of kidney stones: A protocol of systematic review and meta-analysis. Medicine (Baltimore). 99(38): e21910.

19. El Gammal M.Y, Fouda A.A, Meshref AW, Abu-el-Magd AN, Farag FA, et al. (1992) Management of ureteral stones by Extracorporeal Shock Wave Lithotripsy using the Lithostar Lithotripter. J Urol. 148(3 pt 2): 1086-1087.

20. Bierkens AF, Hendrikx AJ, De La Rosette JJ, Stultiens GN, Beerlage HP, et al. (1998) Treatment of mid- and lower ureteric calculi: extracorporeal shock wave lithotripsy vs. Laser 
ureteroscopy. A comparison of costs, morbidity and effectiveness. Br J Urol. 81(1): 31-35.

21. Fetner CD, Preminger GM, Seger J, Lea TA (1988) Treatment of ureteral calculi by extracorporeal shock wave lithotripsy at a multi-use center. J Urol. 139(6): 1192-1194.

22. Netto NR, Lemos GC, Claro JFA (1990) In Situ ESWL for ureteral calculi. J Urol. 144(2): 253-254.

23.
Pettersson B, Tiselius HG (1988) Extracorporeal shock wave lithotripsy of proximal and distal ureteral stones. Eur Urol. 14(3): 184-8.

24. Bach C, Karaolides T, Buchholz N (2011) Shock wave lithotripsy for renal and ureteric stones. Eur Urol Suppl. 2011;10(5): 423432

25. Reynolds LF, Kroczak T, Pace KT (2018) Indications and contraindications for shock wave lithotripsy and how to improve outcomes. Asian J Urol. 5(4): 256-263. 